\title{
Preferencia de oviposición en tres especies de polilla de la papa (Lepidoptera: Gelechiidae)
}

\author{
Mario Andrés Herrera ${ }^{1}$ y Olivier Dangles ${ }^{1,2}$ \\ ${ }^{1}$ Laboratorio de Entomología, Escuela de Ciencias Biológicas Pontificia Universidad Católica del Ecuador, \\ Quito.marioandres45@hotmail.com \\ ${ }^{2}$ IRD, UR 072, Diversity, Ecology and Evolution of Tropical Insects Team, Evolution, \\ Genomes and Speciation Laboratory, UPR 9034, CNRS, 91198 Gif-sur-Yvette Cedex, France and University \\ Paris-Sud 11, 91405 Orsay Cedex, France
}

Recibido: 06, 06, 2012; aceptado: 19, 10, 2012

\begin{abstract}
RESUMEN.- Los insectos plaga son una de las principales causas de pérdidas en cultivos y en almacenes a nivel mundial. En los Andes tropicales, el complejo de polillas de la papa (CPP) (Lepidoptera: Gelechiidae) está conformado por tres especies principales (Tecia solanivora, Symmetrischema tangolias y Phthorimaea operculella) y constituye una de las principales amenazas de los cultivos y almacenes de papas en la región. Se ha demostrado que las interacciones entre las larvas de las tres especies tienen un efecto significativo en la dinámica poblacional del CPP y en los daños causados a los cultivos. Sin embargo, los patrones de interacción inter-especificas durante otras fases del ciclo de vida de estas especies son desconocidos. Este estudio analizó la preferencia de oviposición de hembras del CPP mediante la realización de experimentos en los que hembras del CPP tuvieron que elegir el lugar de oviposición. Hembras de las tres especies del CPP prefirieron ovipositar en tubérculos dañados artificialmente ya que estos podrían ofrecer mejores condiciones para el desarrollo de su descendencia. Cuando hembras del CPP tuvieron que elegir ovipositar entre tubérculos sanos y tubérculos previamente infestados mostraron diferentes comportamientos, encontramos que T. solanivora no tiene sitios de oviposición preferidos mientras que $S$. tangolias y $P$. operculella tienen sitios de oviposición preferidos.
\end{abstract}

PALABRAS CLAVE: Complejo de polillas de la papa, Phthorimaea operculella, preferencia de oviposición, Symmetrischema tangolias, Tecia solanivora.

ABSTRACT.- Insect pests are a major cause of losses in crops and storage globally. In the Tropical Andes, the potato tuber moth (PTM) complex (Lepidoptera: Gelechiidae) is composed of three main species: Tecia solanivora, Symmetrischema tangolias and Pthhorimaea operculella. This complex is a major pest of potato crops and stores in several countries of the Andean region. It has been shown that interactions between larvae of the three species have a significant effect on the population dynamics of the PTM complex and damage to crops. However, patterns of inter-specific interactions during other phases of the life cycle of these species are unknown. This study analyzed the oviposition preference of females of the CPP by conducting two experiments in which females of the CPP had to choose the oviposition site. Females of three PTM complex species showed oviposition preference for artificially damaged tubers over the healthy ones because the damaged tubers may have better conditions for the development of the offspring. When females of the PTM complex had to choose laying eggs between healthy tubers and previously infested ones showed different behaviors, we found that $T$. solanivora have not preferred sites for oviposition while $S$. tangolias and $P$. operculella have preferred oviposition sites.

KEYWORDS: Oviposition preference, Phthorimaea operculella, potato tuber moth complex, Symmetrischema tangolias, Tecia solanivora. 


\section{INTRODUCCIÓN}

El complejo de polillas de la papa (CPP) (Lepidoptera: Gelechiidae), Phthorimaea operculella Zeller, Tecia solanivora Povolny, y Symmetrischema tangolias Gyen constituye una de las principales plagas de este cultivo en la región Andina (Kroschel, 2002). El CPP ataca a los tubérculos en el campo y en el almacén causando grandes pérdidas económicas (Barragán, 2005; Palacios, 1997). Varios trabajos se han enfocado en el estudio del CPP sea en una sola especie o en combinaciones de dos o tres especies, dichos trabajos han descrito y detallado aspectos importantes tales como: identificación, biología y comportamiento, características ecológicas, manejo integrado y diversidad genética (Herrera, 2010). Sin embargo, aún es necesario profundizar los conocimientos existentes en relación a las interacciones intra e interespecíficas que se producen entre las especies del CPP, interacciones entre las que se incluye la preferencia de oviposición.

La localización de hospedero y la oviposición son etapas cruciales en el ciclo de vida de insectos invasivos y pueden jugar un papel muy importante en la competencia entre especies (Broad et al., 2008). Adicionalmente, el comportamiento de oviposición de las hembras determina el ambiente en el cual huevos y posteriormente larvas de desarrollarán (Bonebrake et al., 2010). Finch y Collier, 2003 resumieron en tres pasos básicos la localización de hospedero en insectos: la estimulación química indica cuando empezar; la estimulación visual indica donde empezar y el contacto con el hospedero indica si quedarse o trasladarse. Larvas de varias especies de insectos invasivos no son capaces de trasladarse de una planta a otra y por lo tanto son forzadas a completar su desarrollo en la planta seleccionada por su madre, se cree que las hembras ovipositan en plantas donde su descendencia se desempeñará de la manera más favorable para la especie (Shiojiri et al., 2002). Estudios demuestran que algunas especies prefieren ovipositar en plantas infestadas por individuos de la misma especie y otras prefieren ovipositar en plantas no infestadas (Choh et al., 2008).
Ya que se ha demostrado que el daño producido a los tubérculos es mayor cuando está presente más de una especie de polilla y que la diversidad tiene un efecto significativo en la dinámica poblacional del CPP (Dangles et al., 2009), este estudio tuvo como objetivo determinar sí hembras adultas del CPP prefieren ovipositar en tubérculos que han sido dañados por individuos de una misma especie, en tubérculos que han sido dañados por individuos de otra especie o no discriminan entre tubérculos dañados y tubérculos sanos.

\section{MATERIALES Y MÉTODOS}

En este estudio se analizó la preferencia de oviposición de hembras del CPP mediante la realización de dos experimentos en los que hembras del CPP tuvieron que elegir el lugar de oviposición. Con la primera parte de este estudio evaluamos si las hembras del CPP prefieren ovipositar en tubérculos sanos o tubérculos dañados artificialmente. Para esto se colocó en un recipiente plástico $(3 \mathrm{~L})$ un tubérculo de papa Solanum tuberosum L. V. leona blanca sin infestar y un tubérculo dañado artificialmente [12 orificios de 1 milímetro de grosor y aproximadamente 3 milímetros de profundidad realizados al azar alrededor del tubérculo para simular el daño provocado por las polillas (ver Dangles et al., 2009)]. Para estandarizar el experimento el peso de los tubérculos fue $40 \pm 5 \mathrm{~g}$ (Balanza TU-OI, FA-2104). Posteriormente se colocó en cada recipiente 5 pupas hembra y 5 pupas macho de cada especie del CPP, las pupas de cada especie fueron colocadas en distintos recipientes.

En la segunda parte del estudio evaluamos si hembras del CPP prefieren ovipositar en tubérculos sanos o tubérculos previamente infestados. Se colocó cuatro tubérculos de papa Solanum tuberosum L. v. leona blanca en un recipiente plástico $(3 \mathrm{~L})$. Para estandarizar el experimento el peso de los tubérculos fue $40 \pm 5 \mathrm{~g}$. El primer tubérculo estuvo previamente infestado con 12 larvas de T. solanivo$r a$, el segundo con 12 larvas de $S$. tangolias, el 

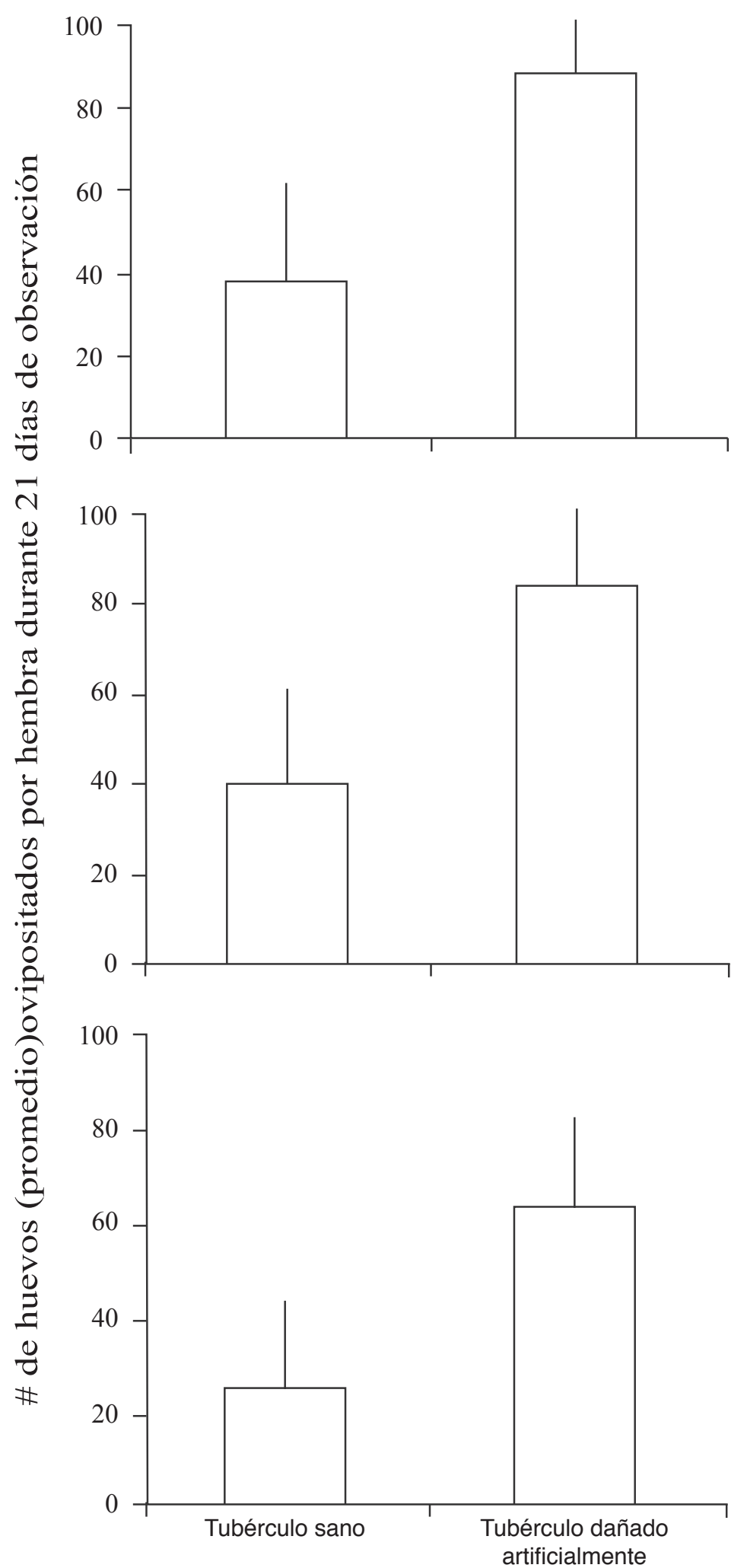

Figura 1. Los gráficos de barras muestran el número de huevos ovipositados (promedio) por hembra del CPP en tubérculos sanos y tubérculos dañados artificialmente durante 21 días. a) T. solanivora Prueba T, $\mathrm{t}=-17.702, \mathrm{p}<0.01, \mathrm{~b}) S$. tangolias Prueba T, $\mathrm{t}=-13.702, \mathrm{p}<0.01, \mathrm{c}) P$. operculella Prueba T, $\mathrm{t}=-10.684, \mathrm{p}<0.01$. Las barras de error muestran el error estándar. tercero con 12 larvas de $P$. operculella (la infestación previa a la colocación de los tubérculos fue de 30 días, la densidad de infestación está de acuerdo a las observaciones realizadas en bodegas de almacenamiento de papas), y el cuarto, un tubérculo sin infestar. Posteriormente, se colocó en cada recipiente los 4 tubérculos descritos anteriormente, y se añadieron 5 pupas hembra y 5 pupas macho de una de las 3 especies; el mismo procedimiento fue utilizado con las otras 2 especies.

Para los dos ensayos el sexo de las pupas fue determinado con la ayuda de un estereomicroscopio (ZEISS, Stemi DV4). Todos los recipientes fueron cerrados con una tapa plástica diseñada para permitir la entrada de aire. Se realizaron 10 repeticiones por cada especie. Una vez que las polillas pasaron del estado de pupa al estado adulto se las dejó en el respectivo recipiente durante 21 días tomando en cuenta la longevidad de los adultos de las polillas (ver Niño, 2004 y Vera Delgadillo et al., 2009). Durante los días que duró el experimento las polillas fueron alimentadas con agua azucarada (solución de sacarosa 2M). Finalmente se realizó el contaje de los huevos (directamente en los recipientes), así como la determinación del lugar en que fueron ovipositados.

Para el primer ensayo los datos obtenidos fueron analizados mediante una prueba $\mathrm{T}$ para muestras independientes y gráficos de barras para cada una de las especies del CPP; y para el segundo ensayo se realizó un ANOVA 

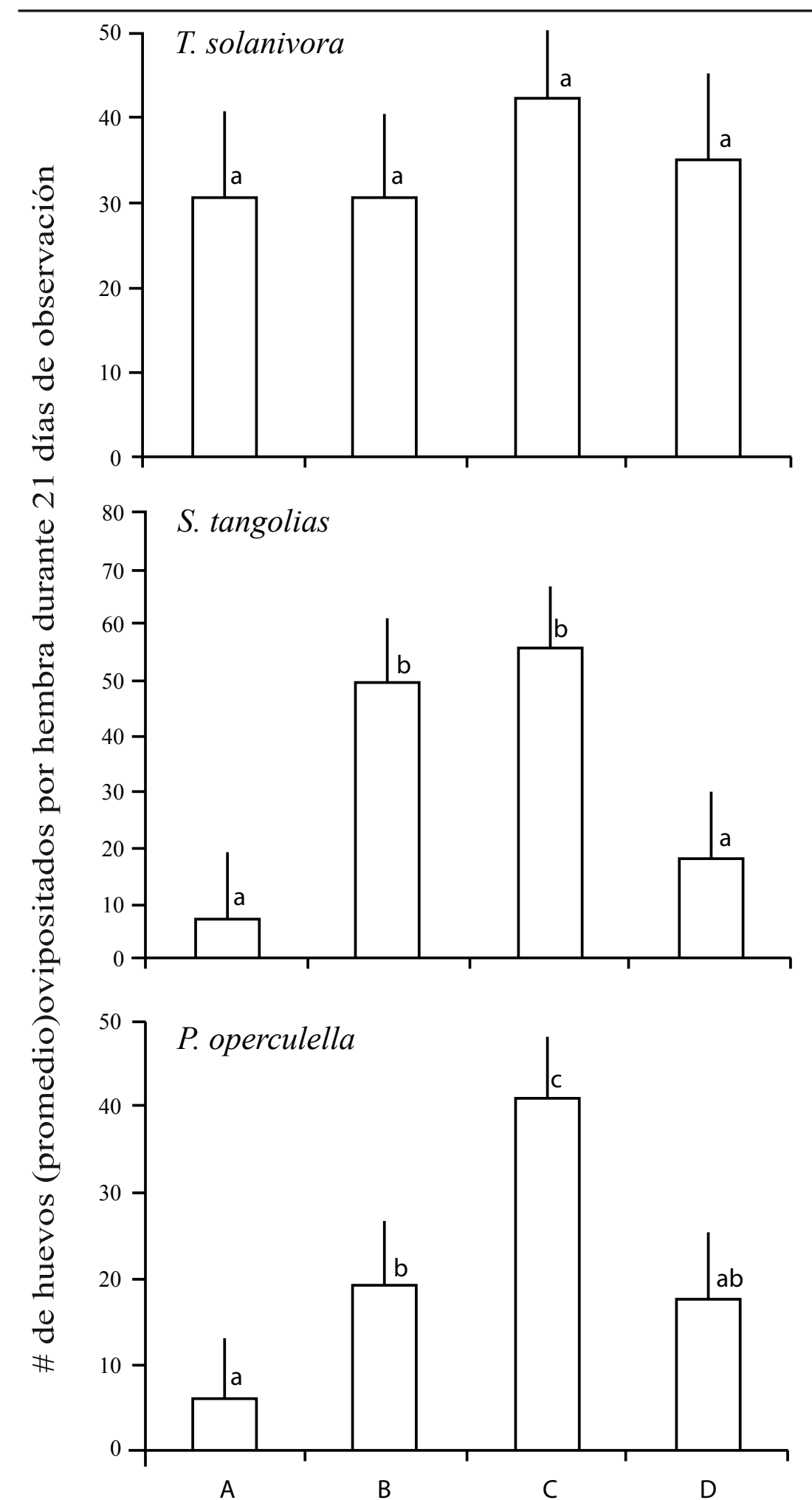

Figura 2. Número de huevos ovipositados (promedio) por hembra del CPP en cuatro tubérculos durante 21 días. A. tubérculo previamente infestado por $T$. solanivora, B. tubérculo previamente infestado por $S$. tangolias, C. tubérculo previamente infestado por $P$. operculella, D. tubérculo sano. Letras distintas muestran que hay diferencias significativas entre los sitios de oviposición (Prueba de Tukey, $\alpha=0.05$, T. solanivora, $\mathrm{p}=0.27, S$. tangolias, $\mathrm{p}<$ 0.01$, P. operculella, $\mathrm{p}<0.01)$. Las barras de error muestran el error estándar.

de una vía, una prueba de Tukey para determinar los rangos de significación entre los sitios de oviposición y un gráfico de barras para cada especie del CPP. Los análisis fueron realizados con el paquete informático PASW Statistics 18.

\section{RESULTADOS}

Preferencia de oviposición en tres especies del CPP entre tubérculos sanos y tubérculos dañados artificialmente.- Los resultados obtenidos en los gráficos de barras (Figura 1) y confirmado mediante los respectivas Pruebas de $\mathrm{T}$ mostraron que las hembras de las tres especies del CPP prefieren ovipositar en tubérculos dañados artificialmente que en tubérculos sanos. ( $T$. solanivora Prueba $\mathrm{T}, \mathrm{t}=-17.702, \mathrm{p}<$ 0.01, S. tangolias Prueba $\mathrm{T}, \mathrm{t}=-13.702, \mathrm{p}<0.01 \mathrm{y}$ P. operculella Prueba $\mathrm{T}, \mathrm{t}=$ $-10.684, \mathrm{p}<0.01)$.

Preferencia de oviposición en tres especies del CPP entre tubérculos sanos y tubérculos previamente infestados.- En hembras de $T$. solanivora la diferencia entre los sitios de oviposición no fue significativa (Figura 2, Prueba de Tukey, $\alpha=0.05, p=0.27$ ). En contraste, hembras de $S$. tangolias prefieren ovipositar en tubérculos infestados por individuos de la misma 
especie y tubérculos infestados por $P$. operculella que en tubérculos infestados por $T$. solanivora y tubérculos sanos (Figura 2, Prueba de Tukey, $\alpha=0.05, \mathrm{p}<0.01)$ y hembras de $P$. operculella prefieren ovipositar en tubérculos infestados por individuos de la misma especie que en tubérculos infestados por T. solanivora o infestados por $S$. tangolias y tubérculos sanos (Figura 2, Prueba de Tukey, $\alpha=0.05, \mathrm{p}<$ $0.01)$.

\section{DISCUSIÓN}

La primera parte de este estudio muestra que hembras de las tres especies del CPP prefieren ovipositar en tubérculos dañados artificialmente que en tubérculos sanos. Se conoce que las plantas producen compuestos volátiles para defenderse de insectos plaga y que la mezcla de estos compuestos depende del comportamiento alimenticio de los insectos pero también que adultos de insectos herbívoros son atraídos hacia la planta hospedera por compuestos volátiles emanados por ésta (Broad et al., 2008; Liu et al., 2005). Aunque en este estudio solo se trabajó con tubérculos, posiblemente estos produjeron compuestos volátiles que atraen a insectos en mayor cantidad que aquellos compuestos que los repelen. Debido a que no existe información del efecto de compuestos volátiles en el comportamiento de adultos del CPP una hipótesis es que las hembras del CPP prefirieron ovipositar en tubérculos dañados artificialmente por que estos presentaban mejores condiciones para la supervivencia de la progenie, específicamente los orificios realizados alrededor de los tubérculos pudieron haber influido en la elección de las hembras mediante la facilitación para la entrada de las larvas.

La segunda parte del estudio muestra que la preferencia de oviposición es distinta para las tres especies del CPP cuando tienen que elegir entre tubérculos infestados y tubérculos sanos. Aparentemente hembras de $T$. solanivora no discriminan sus sitios de oviposición, hembras de $S$. tangolias prefieren ovipositar en tubérculos infestados por individuos de su misma especie y en tubérculos infestados por $P$. operculella y hembras de $P$. operculella prefieren ovipositar en tubérculos infestados por individuos de su misma especie. Posiblemente la preferencia de oviposición en hembras del CPP esté relacionada con la hipótesis de preferencia-desempeño. Esta hipótesis establece que las hembras de insectos herbívoros deberían preferir ovipositar en plantas en las que las larvas aseguren su desempeño (desarrollo y supervivencia). Varios estudios se han enfocado en probar la hipótesis de preferencia-desempeño (Mphosi y Foster, 2010; Choh et al., 2008; Shiojiri et al., 2002; Harris et al., 2001). Es posible que la hipótesis de preferencia-desempeño se cumpla en dos de las tres especies del CPP, $S$. tangolias y $P$. operculella, ya que estas dos especies provocan menor daño a los tubérculos en comparación a $T$. solanivora; por lo que evidentemente una vez que las larvas eclosionen tendrían una mejor fuente de alimento y por lo tanto podrían asegurar el éxito de su ciclo vital. Además, como muestran los resultados tanto $S$. tangolias como $P$. operculella tienen sus tasas de oviposición más bajas en los tuberculos infestados por $T$. solanivora, hecho que confirmaría la hipótesis.

En contraste, también hay estudios que muestran que en varias especies de insectos herbívoros la relación entre preferencia de oviposición y desempeño de larvas es muy baja (Müller y Arand, 2007; Scheirs y De Bruyn, 2002); esto podría explicar la preferencia de oviposición en $T$. solanivora ya que como se muestra en el estudio hembras de esta especie no tienen un lugar de oviposición preferido. Ya que en este estudio solo se evaluó la preferencia de oviposición, estudios complementarios deberían enfocarse en el desempeño larval, es decir, tomar en cuenta la tasa de supervivencia, la tasa de consumo y la fecundidad de las larvas que llegan a completar el ciclo de vida para de esta manera evaluar la hipótesis de preferencia-desempeño. 


\section{AGRADECIMIENTOS}

La realización de este trabajo fue posible gracias a la colaboración de la Pontificia Universidad Católica del Ecuador (PUCE), el Instituto de Investigación para el Desarrollo (IRD) y la Fundación McKnight en el proyecto Enfoques Innovativos para el Manejo Integrado de Plagas en los Andes, Beca No. 09-022.

\section{REFERENCIAS BIBLIOGRÁFICAS}

Barragán A. 2005. Identificación, biología y comportamiento de las polillas de la papa en el Ecuador, Boletín PROMSA, MAGPUCE, Quito, Ecuador.

Bonebrake TC, Boggs CL, McNally JM, Ranganathan J y Ehrlich PR. 2010. Oviposition behavior and offspring performance in herbivorous insects: consequences of climatic and habitat heterogeneity. Oikos, 119: 927-934.

Broad ST, Schellhorn NA, Lissont SN y Mendham NJ. 2008. Host location and oviposition of lepidopteran herbivores in diversified broccoli cropping systems. Agricultural and Forest Entomology, 10: 157-165.

Choh Y, Uefune M y Takabayashi J. 2008. Diamondback moth females oviposit more on plants infested by non-parasitised than by parasitized conspecifics. Ecological Entomology, 33: 565-568.

Dangles O, Mesías V, Crespo-Pérez V y Silvain JL. 2009. Crop damage increases with pest species diversity: evidence from potato tuber moths in the tropical Andes. Journal of Applied Ecology, 1: 1-7.

Finch S y Collier RH. 2003. Insects can see clearly now the weeds have gone. Biologist, 50: 132-135.

Harris MO, Sandanayaka My Griffin W. 2001. Oviposition preferences of the Hessian fly and their consequences for the survival and reproductive potential of offspring. Ecological Entomology, 26: 473-486.

Herrera DMA. 2010. Interacciones intra e inter-específicas entre polillas de la papa (Lepidoptera: Gelechiidae). Tesis de Licenciatura, Pontificia Universidad Católica del Ecuador. Quito, Ecuador.
Kroschel J. 2002. Desafíos y oportunidades para el manejo de plagas en papa en países en desarrollo. Boletín de la papa CIP. Lima, Perú.

Liu SS, Li YH, Liu YQ y Zalucki MP. 2005. Experience-induced preference for oviposition repellents derived from a nonhost plant by a specialist herbivore. Ecology Letters, 8:722-729.

Mphosi MS y Foster SP. 2010. Female preference and larval performance of sunflower moth, Homoeosoma electellum, on sunflower pre-breeding lines. Entomologia Experimentalis et Applicata, 134: 182-190.

Müller C y Arand K. 2007. Trade-offs in oviposition choice? Food-dependent performance and defence against predators of a herbivorous sawfly. Entomologia Experimentalis et Applicata, 124: 153-159.

Niño L. 2004. Revisión sobre la Polilla de la Papa Tecia solanivora en Centro y Suramérica. Suplemento Revista Latinoamericana de la Papa, 18 pp.

Palacios M. 1997. Manual de Capacitación Principales Plagas de la Papa: La Polilla de la Papa y La Mosca Minadora. Centro Internacional de la papa (CIP) Fascículo 3.7. Lima, Perú.

Scheirs J y de Bruyn L. 2002. Integrating optimal foraging and optimal oviposition theory in plant-insect research. OIKOS, 1: 187-191.

Shiojiri K, Takabayashi J, Yano S y Takafuji A. 2002. Oviposition preferences of herbivores are affected by tritrophic interaction webs. Ecology Letters, 5: 186-192.

Vera Delgadillo V, Gonzales Aldana MA, Chambilla Quisbert C y Garret K. 2009. Efecto de las variaciones climáticas en el comportamiento de dos polillas (Phthorimaea operculella y Symmetrischema tangolias) en el cultivo de papa en comunidades del Altiplano Central. Sustainable Agriculture and Natural Resource Management Collaborative Research Support Program (SANREM CRSP). Working Paper No. 01-09. 